\title{
A Reply to Pazzani's Book Review of "Inductive Logic Programming: Techniques and Applications"
}

\author{
VALA LAVRAC \\ SASO DZEROSKI \\ 1. Stefan Institute, Jamova 39.61000 Ljubtjana, Sloveria \\ Editor: Alberto Segre
}

nada.lavrac (dijs.si

Sako.drerosk1 (di) ys.st

\section{Introduction}

We are pleased to reply to Michael Pazzani's thorough review of our book on Inductive I ngir. Programming (II P) The bonk gives an introduction to this now and fast growing tield. We would like to emphasize that it also gives an in-depth account of the most established and applicable techniques within the field and several applications of these techniques. Our reply presents the view we trok when writing the hook; this ancwers most of the specific points made by the reviewer.

Inductive I oygic Programming (ILP) is concerned with learning first-order rules formulated in the language of logic programs. ILP systems use this language for representing background knowledge, examples and hypotheses. The main motivation for using this language is its clear syntax and semantics, as well as the sound theorctical and practical methods for deductive inference in it. Tarly relational learning sycirms, wh: as ARCHES, INDUCE, and ML-SMAR', which use other representation formalisms, are not generally considered II.P systems. Thus, they are not discussed in the book

We have consciously biased the book toward II.P rechniques and systems that have reached a certain degree of applicability to practical problems. This has strongly infloenced the choice of topics covered by the book. For example, while learning recursive rules (inverted implication) and predicate invention have received a lot of attention within the IL.P community, few practical results exist so far. Furthermore, while karnability results are important, they are not of immediate practical interest. Consequently, we have decided not to discuss, learnability, including, for example, the learnability results obtained by transtorming ILP problems to propositional lorm (Dzeroski et al., 1992).

The practical orientation of the book explains the omission of several techniques and systems montioned in the review, including interactive ILP isystems (c.g.. CINT). AI though the first II P systems were of an interactive nature and important developments in this area have been made, few interactive II.P systems have been applied to practical problems. Interaction with the user (oracle) is in fact demanding and so are the practicul applications of interactive II.P systems. The empirical II P setting, on the other hand, resembles the well-understood propositional learning setting as used in the widely used ID3 and $A Q$ systeus. In short, nost existing II P applications involvo compirical II P systems; hence the focus of our book is on empirical IL.P. 
In addition to giving an introduction to $\mathrm{ILP}$, the book is also an in-depth study on multi-class learning and the handling of real numbers, imperfect data and irrelevant features, which are centra! topies of our own ILP rescarch. 'These lechniques are embedded in the ILP systems LINUS, DINUS, and mFOIL. Our research has focused on adapting techniques from propositional learning for use in II.P. Transforming an II.P problem to propositional torm (as done in LINUS and DINUS) enables the use of a variety of propositional learning techniques. Moreover, these techniques can also be adapted for direct use in ILP systems (e.g., for noise-handling in mFOII). In the transformation approach, background knowledge (In the lorm of a logic program) is used to introduce new attributes. Although this may look simple (as in the medical application), it suffices for learning the important class of determinate logie programs, which is also the hypothesis language of GOLEM (Muggleton \& Feng 1990). Besides its practical applicability, the transformation approach is important for understanding the relation of propositional learning to II P and the computational complexity of learning.

Although many ILP applications are still proof-of-the-principle applications, some are getting closer to real use. The accuracies achieved in the finite element mesh design domain are, in tact, much higher now (Dolsak ot al., 1994) than originally reported in the book. The molecular biology ILP applications by Stephen Muggleton and his colleagues (Muggleton et al., 1992; Srinivasan et al., 1994) are important in the sense that they have produced new knowledge published in top scientific journals in the respective application areas. Two ol these applications, drug design and protein secondary structure prediction, are summarized in the book. The recent application to predicting mutagenicity of chemical compounds from their chemical structures is especially noteworthy, as this is a problem that can not be addressed by either propositional systems or determinate II.P systems (Srinivasan et al., 1994). We are pleased to observe that applications more interesting than those presented in the book are emerging every day.

The lack of a conclusion chapter that would recommend particular ILP systems for particular problem classes and give information on how to obtain ILP systems is partly due to the fact that IL.P is a young and fast-growing field. It is difficult to make definite conclusions and recommendations about which algorithm is appropriate for which class of problems; this is a difficult task even for the propositional learning area, which has been active for a long time. While few II.P systems were publicly available at press time, scveral are now available via anonymous ftp. ${ }^{\text {' }}$

We believe that despite its inevitably incomplete coverage of the freld, our hook is a timely introductory text that contributes to the development of II P and its accessibility to the outside world.

\section{Notes}

I. ILP sofiware datasets, and publications can be obtained by anonymous ftp from Bomn Germany at

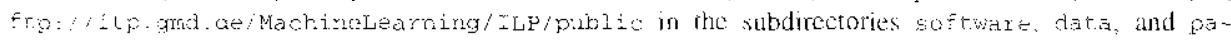
pars. Some can alwo be found in the UCl Machine learning Repository. Additional intormation on ILP research wan be accessed through the World Wide Web server located in Ljubljana. Slovenia al r...tp: / www.

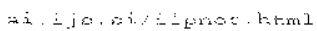




\section{References}

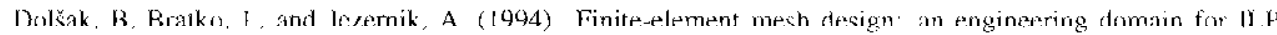
application. In S. Wrobel (Ed.) Proc. Fourh Int. Workshop on Inductive Logic Programming (11.P.94) (pp. 305-320) Bad Honnef/Bonn. GMD Studien Number 237. Bonn: GMD.

Džeroski, S., Muggleton. S., and Russell, S. (1992). PAC-teamability of determinate logic programs. In Proc

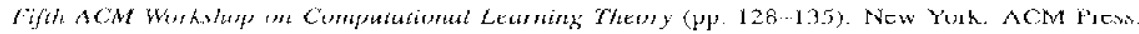

Muggleton, S., and Feng, C. (1990). Efficient induction of logic programs. In Proc. First Conference un Algorithmic Learning Theory (pp. 368 381) Ohmsha, Tokyo.

Muggleton, S., King, R.D., and Sternberg, M.J.E. (1992). Protein secondary structure prediction using logic: Frorein tingtneering, $3,04 /-05 \%$

Srinivasan, A., Mugglaton, S.H., King, R.D., and Sternberg, M.J.F. (1994). Mutagenesis: II.P experiment: in a non-determinate biological domain. In $\mathrm{S}$. Wrobel (Ed.) Pros. Fourth Int. Workshop ort Inductive Iogit. Programming (17.P-94) (pp. 217 232) Bad Honnef/Bonn. GMD Siudien Number 237. Bonm: (iMD). 